\title{
Slow Dynamics in Ion-Conducting Sodium Silicate Melts: Simulation and Mode-Coupling Theory
}

\author{
Th. Voigtmann ${ }^{1}$ and J. Horbach ${ }^{2}$ \\ ${ }^{1}$ University of Edinburgh, School of Physics, JCMB The Kings Buildings, Mayfield Road, Edinburgh EH9 $3 J Z$, Scotland \\ ${ }^{2}$ Institut für Physik, Johannes-Gutenberg-Universität Mainz, Staudinger Weg 7, D-55099 Mainz, Germany
}

(Dated: November 12, 2018)

\begin{abstract}
A combination of molecular-dynamics (MD) computer simulation and mode-coupling theory (MCT) is used to elucidate the structure-dynamics relation in sodium-silicate melts (NSx) of varying sodium concentration. Using only the partial static structure factors from the MD as an input, MCT reproduces the large separation in relaxation time scales of the sodium and the silicon/oxygen components. This confirms the idea of sodium diffusion channels which are reflected by a prepeak in the static structure factors around $0.95 \AA^{-1}$, and shows that it is possible to explain the fast sodium-ion dynamics peculiar to these mixtures using a microscopic theory.
\end{abstract}

PACS numbers: 64.70.Pf, 61.20.Ja, 66.30.Hs

Glass-forming mixtures of $\mathrm{SiO}_{2}$ with an alkali oxide such as $\mathrm{Li}_{2} \mathrm{O}, \mathrm{Na}_{2} \mathrm{O}$, or $\mathrm{K}_{2} \mathrm{O}$ are of general interest for the study of transport mechanisms in amorphous condensed matter. They all show alkali ion mobility that is much higher, often by orders of magnitude, than that of the silicon and oxygen atoms forming a tetrahedral network structure. More than 20 years ago, the idea of preferential diffusion pathways in the $\mathrm{Si}-\mathrm{O}$ network was proposed as an explanation for the fast transport of alkali ions [1, 2]. A recent study of sodium silicate melts using a combination of molecular dynamics (MD) computer simulations and inelastic neutron scattering [3] has shown that indeed percolating sodium-rich channels are formed in the intermediate-range static structure. The sodium ions perform a hopping motion along those channels [4], and simulation studies on different alkali silicates have identified various properties of the characteristic alkali ion sites involved in this motion $[\underline{5}, 6,6,6,8]$.

If the presence of intermediate-range order in the static structure is intimately related to the high mobility of the alkali ions, it should be possible to predict the dynamics, in particular the time-scale separation between the sodium transport and the silicon/oxygen "matrix" dynamics, from this static structure. Such a structuredynamics relation is, however, not easily formulated in terms of a microscopic theory. Here, we discuss a theoretical framework in which this is possible.

The mode-coupling theory of the glass transition (MCT) 9], has been formulated to provide a firstprinciples description of slow dynamics in glass-forming liquids. It needs as input the static equilibrium information about a system, in its most common form only the partial static structure factors $S_{\alpha \beta}(q)$. The MCT equations then yield dynamic correlation functions, in particular the normalized dynamic structure factors, $F_{\alpha \beta}(q, t)=S_{\alpha \beta}(q, t) / S_{\alpha \beta}(q)$ (also called the coherent intermediate scattering functions), and the incoherent self-intermediate scattering functions of a given species $\alpha, F_{\alpha}^{s}(q, t)$. They can be directly compared to scatter- ing experiments or simulation results and determine, via Green-Kubo relations, the transport coefficients.

The programme of predicting, using MCT, dynamical features from the equilibrium structure of the liquid and testing the predictions against MD simulations, has proven successful for some paradigmatic dense liquids like the hard-sphere system [10], binary mixtures of hard spheres 11], Lennard-Jones particles [12, 13], a model of $\mathrm{NiZr}_{4}$ 14], and models of more complicated dense glass formers 15, 16, 17]. In these systems, the agreement was qualitative and often even quantitative. For a computermodeled silica melt, MCT was able to predict the form factor of the glass, i.e. the height of the plateau in the dynamic structure factors, qualitatively, and even to within a few percent if in addition to $S_{\alpha \beta}(q)$ static triplet correlations were taken into account 18 .

In this work, the MCT equations are solved for sodium silicates, using MD-simulated static structure factors as an input. The resulting dynamic structure factors are compared to the ones obtained from MD. We demonstrate that, at least on a qualitative level, the theoretical framework of MCT allows to describe the large timescale separation in sodium silicates. This establishes a structure-dynamics relation based on static pair correlations. It confirms that the high mobility of sodium ions in sodium silicates is indeed intimately related the presence of intermediate range order (i.e. the aforementioned channel structure) as reflected by a prepeak in partial static structure factors.

MD simulations were performed for sodium silicates with different compositions, $\left(\mathrm{Na}_{2} \mathrm{O}\right)\left(\mathrm{SiO}_{2}\right)_{x}[\mathrm{NS} x]$, with $x=2,3,5,20$, and 40 . The simulations were all done at constant density $\rho=2.37 \mathrm{~g} / \mathrm{cm}^{3}$, using $N=8064$ particles and a slight modification of the pair potential by Kramer et al. 19], which is based on ab initio calculations. For details, we refer to Ref. 20, 21. Based on the thus obtained $S_{\alpha \beta}(q)$ for NS2 and NS20, the MCT equations of motion for $S_{\alpha \beta}(q, t)$ 22] were solved numerically as outlined in Ref. 23], with discrete wave numbers 


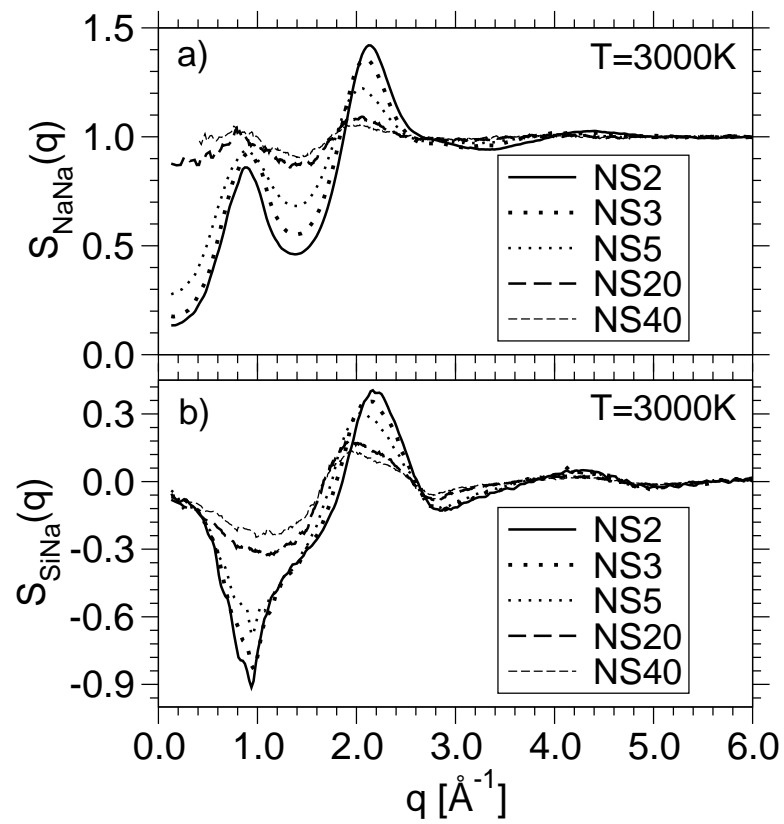

FIG. 1: Partial structure factors for the indicated sodium silicates at $T=3000 \mathrm{~K}$, obtained from MD simulations. a) $S_{\mathrm{NaNa}}(q)$, b) $S_{\mathrm{SiNa}}(q)$.

$0.1 \AA^{-1} \leq q<20 \AA^{-1}$ of step size $\Delta q=0.1 \AA^{-1}$, applying a five-point running average to the input. The MCT solutions are fully determined only through $S_{\alpha \beta}(q)$ and the static triplet correlation function $c_{\alpha \beta \gamma}^{(3)}(\vec{q}, \vec{k})$. We set the latter to zero thus approximating the three-point correlations by a convolution approximation.

The structure factor input is exemplified in Fig. [ where we plot $S_{\mathrm{NaNa}}(q)$ and $S_{\mathrm{SiNa}}(q)$ for different sodium concentrations at the temperature $T=3000 \mathrm{~K}$. Note that even at this high temperature, typical "matrix" relaxation times are already in the range of $10-100 \mathrm{ps}$. The channel structure formed by the alkali ions on intermediate length scales, at about $6-7 \AA$, is clearly visible as a prepeak around $q=0.95 \AA^{-1}$. This prepeak becomes more intense with increasing sodium concentration, and, remarkably, its location does not change significantly over the whole considered sodium concentration range. This is in agreement with our recent finding that the prepeak reflects a characteristic intermediate-range chemical ordering of sodium that leads to the formation of sodiumdiffusion channels. However, in Fig. 1 no obvious scale separation hints towards the peculiar sodium dynamics. In MCT for single-component systems, relaxation times are typically correlated with peaks in $S(q)$; thus, from a peak in the diagonal element $S_{\alpha \alpha}(q)$, one might expect a slowing down of the dynamics around the prepeak position, but nothing particular for the self-relaxation times.

In the comparison of dynamical quantities between MD and MCT, one has to note that the theory predicts a divergence of relaxation times at $T=T_{c}$ : the idealized

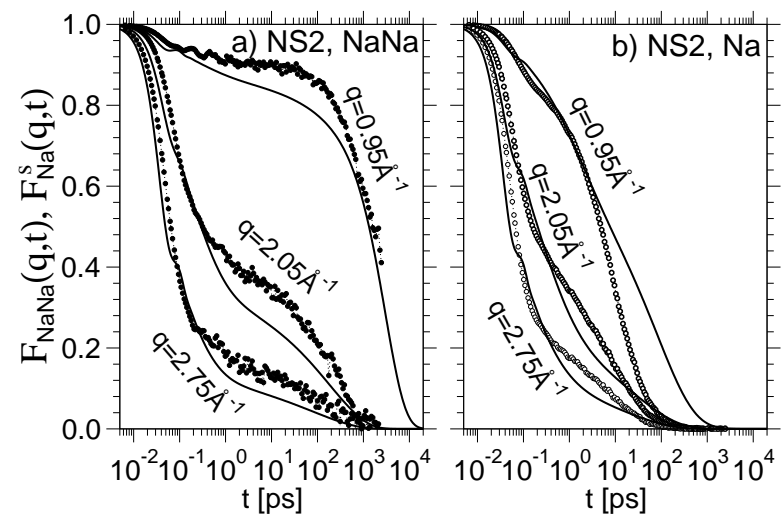

FIG. 2: Normalized sodium-sodium intermediate scattering functions, $F_{\mathrm{NaNa}}(q)$ (left panel), and sodium-self intermediate scattering functions, $F_{\mathrm{Na}}^{s}(q, t)$ (right panel), for sodium disilicate (NS2). The symbols are MD results for $T=2100 \mathrm{~K}$ (from Ref. 4]); the solid lines are MCT results for $T_{\mathrm{MCT}}=3410 \mathrm{~K}$; $q$ values are indicated.

glass transition, which is smeared out in experiment. A scaling analysis of the MD data gives a value for $T_{c}$, which is lower than the value calculated within the theory. Hence, in order to compare dynamical data that show the same degree of slowing down, i.e. the same separation between the structural relaxation time scale and the time scale of molecular short-time motion, we compare MD data for temperature $T$ with MCT data at a different (higher) temperature $T_{\mathrm{MCT}}$. This is then the only free parameter in our comparison.

Note that, despite the quantitative error in the value of $T_{c}$, the qualitative change of $T_{c}$ with varying sodium concentration is captured by MCT: from a scaling analysis of the simulation data, one gets $T_{c} \approx 3330 \mathrm{~K}$ for silica 24 and $2000 \mathrm{~K}$ for NS2 25. The respective values we calculate within the theory are $T_{c} \approx 3983 \mathrm{~K}$ and $3105 \mathrm{~K}$.

The large difference in time scales between the matrix relaxation and that of sodium transport, on almost all length scales, is best evidenced in the sodium-sodium (coherent) and the sodium-self (incoherent) correlation functions [4], $F_{\alpha \alpha}(q, t)=S_{\alpha \alpha}(q, t) / S_{\alpha \alpha}(q)$ and $F_{\alpha}^{s}(q, t)$ in the case $\alpha=\mathrm{Na}$. The former follows the slow matrix relaxation, while the latter decays much faster, giving rise to fast sodium diffusion [4]. MD results at $T=2100 \mathrm{~K}$ together with MCT results at $T_{\mathrm{MCT}}=3410 \mathrm{~K}$ for the two quantities at selected wave numbers $q$ are shown in Fig. 2 We have chosen the latter temperatures for the comparison, since at these temperatures the coherent relaxation times as obtained from MD and MCT are very similar at $q=0.95 \AA^{-1}$ (see also below). At this wave number, the time-scale separation is most pronounced: while the sodium-sodium correlations decay at $\tau \approx 10^{4} \mathrm{ps}$, the sodium-self correlations decay at $\tau^{s} \approx 10^{2} \mathrm{ps}$, i.e. two orders of magnitude faster. MCT underestimates this separation, but it still gives a factor of about 50 between the 


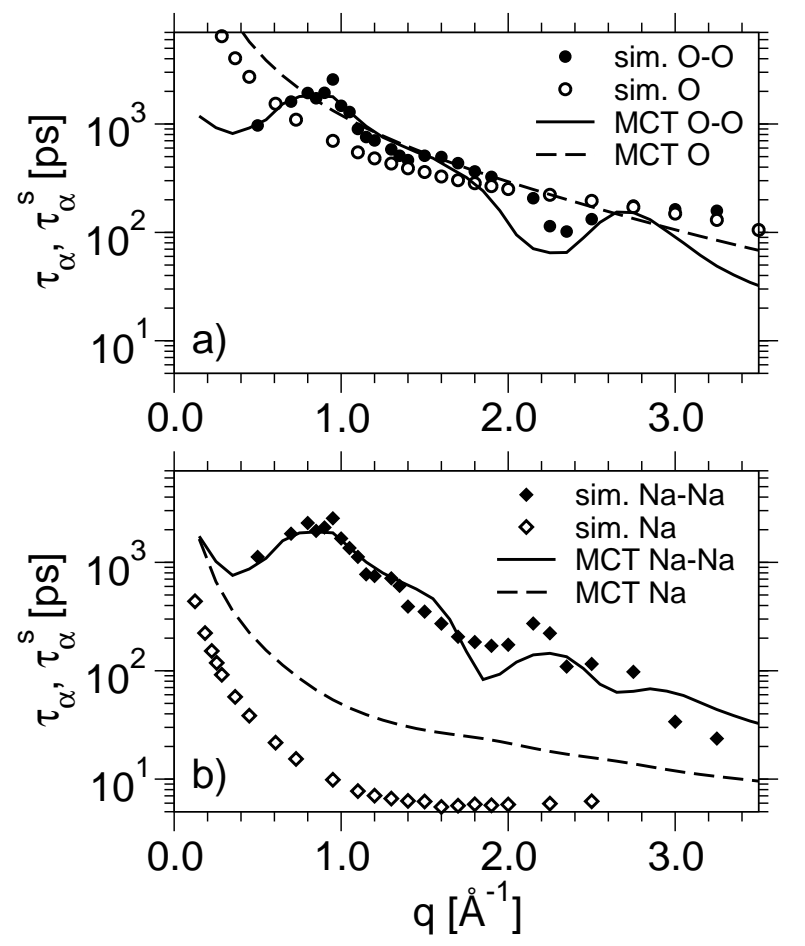

FIG. 3: Structural relaxation times $\tau_{\alpha}(q)$ for coherent density relaxation functions $F_{\alpha \alpha}(q, t)$ and $\tau_{\mathrm{Na}}^{s}(q)$ for the incoherent density relaxation function $F_{\mathrm{Na}}^{s}(q, t)$, a) $\tau_{\mathrm{O}}(q)$ and $\tau_{\mathrm{O}}^{s}(q)$, b) $\tau_{\mathrm{Na}}(q)$ and $\tau_{\mathrm{Na}}^{s}(q)$. As indicated, symbols are simulation results $(T=2100 \mathrm{~K})$ and lines are MCT results $(T=3410 \mathrm{~K})$. See text for the definition of $\tau_{\alpha}(q)$ and $\tau_{\alpha}^{s}(q)$.

two time scales. Note that in the hard-sphere system, the coherent and incoherent relaxation times agree within a factor of 4 at comparable intermediate $q$. Hence, the theory captures the peculiarity of the fast sodium dynamics in NS2 qualitatively correctly. At the higher wave number $q=2.05 \AA^{-1}$, the time-scale separation is smaller, but still $\tau / \tau^{s} \approx 20$ for the MD data. The MCT result, $\tau / \tau^{s} \approx 14$, here is even closer to the simulation value.

Overall, the theory reproduces well the qualitative facts seen in the MD simulations. There are two major types of discrepancy visible: first, the heights of the plateaus seen in the correlation functions at intermediate times differ. They are connected to the glass form factors, for which in the case of silica [18] the agreement was quantitative with static triplet correlations taken into account, but qualitative only without them (as in our case). We therefore expect this first problem to be curable by providing better static structure input. The second problem is the shape of the correlators, especially the sodium-self correlators at long times: for example, for $q=0.95 \AA^{-1}$, MCT predicts a long, extremely stretched decay from 0.6 to zero over more than 2 decades in time, which is not observed in the simulation.

To test the wave-vector dependence of the dynamics in more detail, we turn to a discussion of structural

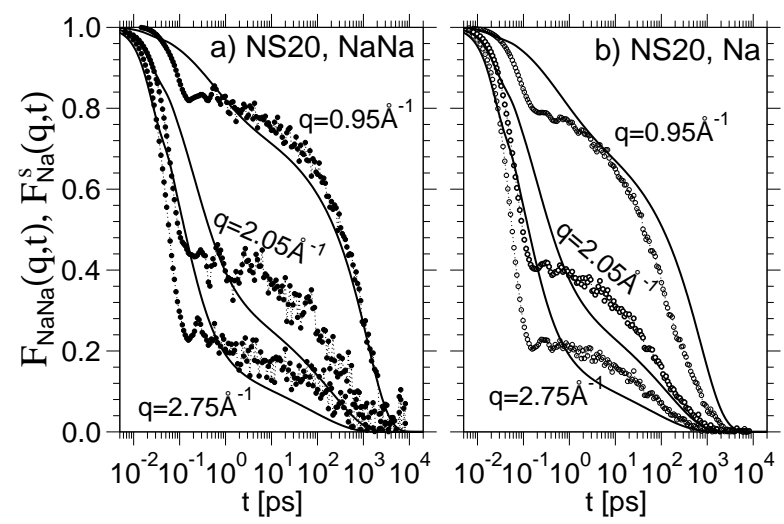

FIG. 4: Normalized sodium-sodium intermediate scattering functions, $F_{\mathrm{NaNa}}(q)$ (left panel), and sodium-self intermediate scattering functions, $F_{\mathrm{Na}}^{s}(q, t)$ (right panel), as in Fig. 2 but for NS20. MD results (symbols, from Ref. [26]) are for $T=$ $2500 \mathrm{~K}, \mathrm{MCT}$ results (solid lines) for $T_{\mathrm{MCT}}=4000 \mathrm{~K}$.

relaxation times for the coherent, $\tau_{\alpha}(q)$, and the incoherent correlators, $\tau_{\alpha}^{s}(q)$. We define them through fits of stretched exponentials to the correlation functions, $F_{\alpha \alpha}(q, t) \approx A_{\alpha}(q) \exp \left[-\left(t / \tau_{\alpha}(q)\right)^{\beta_{\alpha}(q)}\right]$ at long times, with $\beta_{\alpha}(q)<1$ and $A_{\alpha}(q)<1$, and similarly for $F_{\alpha}^{s}(q, t)$. This yields a reasonable description of the dynamics for $t \gtrsim 1$ ps. In Fig. [3 coherent and incoherent relaxation times are plotted for oxygen and sodium. MD and MCT results are shown as symbols and lines, respectively. The coherent relaxation times are, at fixed $q$, all of the same order of magnitude, and they almost coincide around $q \approx 1 \AA^{-1}$, the region corresponding to the main structural features of the system (note that this holds also for $\left.\tau_{\mathrm{Si}}(q)\right)$. The values obtained from MCT and those from MD are in good agreement given that we only have one adjustable parameter. In particular, their subtle $q$ dependence is reproduced. The oxygen-self relaxation time $\tau_{\mathrm{O}}^{s}(q)$ is of the same order as the corresponding coherent one, $\tau_{\mathrm{O}}(q)$, except for $q \rightarrow 0$. This is what one expects in a typical glass-forming liquid. On the contrary, the sodium-self relaxation time $\tau_{\mathrm{Na}}^{s}(q)$ is much smaller than all other structural relaxation times, both in the MCT and the MD results. Even if the MCT results for this quantity are systematically higher than the MD data (reflecting the difference in shape of the sodium self-correlators in Fig. 22), this confirms that MCT indeed predicts the fast-ion dynamics in NS2 from the equilibrium structure alone.

We now investigate the effect of sodium concentration on the dynamics by discussing simulation and MCT results for NS20. Fig. 团 shows the coherent and incoherent sodium-density correlators for this system, similar to the NS2 results shown in Fig. 2] For NS20, results at higher $T$ than in NS2 are shown, in order to compare states which show the same degree of slowing down, i.e. $\tau_{\mathrm{Na}}=\mathcal{O}\left(10^{3} \mathrm{ps}\right)$ for $q \approx 0.95 \AA^{-1}$. The main effect of 
reducing the sodium concentration from NS2 to NS20 on the dynamics is that the sodium-self relaxation now is appreciably slower, as is evident from the right-hand panel in Fig. 4. The relaxation time obtained from the MD data is $\tau_{\mathrm{Na}}^{s}\left(q=0.95 \AA^{-1}\right) \approx 10^{3} \mathrm{ps}$, similar to the $\tau_{\mathrm{Na}}(q)$ value at that $q$, i.e. all relaxation times, including that of the sodium-self correlations, are now of the same order of magnitude at fixed $q$. MCT again somewhat overestimates the sodium-self relaxation times, but the qualitative sodium-concentration effect is well explained by the theory.

Also for NS20, the shape of the relaxation curves predicted by MCT for the sodium-self correlators disagrees with that observed in MD. But here, the discrepancy is found largely in the form of the relaxation towards the plateau, while the further deviations visible in Fig. 4 are ascribable to the error in those plateau values as explained above. The observation of deviations at relatively short times, compared to the structural relaxation time, is typical for comparisons between MCT and Newtonian dynamics MD simulations [1, 12].

In summary, we have used MCT in conjunction with computer-simulated static structure factors for different sodium silica melts. The theory is able to explain the decoupling of time scales between the sodium-self dynamics and the much slower network relaxation, as well as the dependence of this decoupling on the sodium concentration. The ratios of relaxation times are in qualitative agreement with the MD results. Since MCT only makes use of the partial structure factors $S_{\alpha \beta}(q)$, this shows that indeed a structure-dynamics relation holds for the fast ion transport in these alkali silicates, and that MCT provides a possible microscopic explanation for it. The description is not yet on a quantitative level, but one can expect that taking into account more detailed information about the equilibrium static structure, i.e. static triplet correlations, will improve on at least some of the deviations pointed out above.

We thank M.E. Cates, W. Götze, W. Kob, and W. Paul for their comments on the manuscript. The authors are supported through the Emmy Noether program of the DFG, grants Ho 2231/2-1/2 (J.H.) and Vo 1270/11 (Th.V.), and EPSRC grant GR/S10377/01 (Th.V.). Computing time on the JUMP at the NIC Jülich is gratefully acknowledged.

[1] C. A. Angell, P. A. Cheeseman, and S. Tamaddon, J. Phys. (France) C9-43, 381 (1982).
[2] G. N. Greaves, J. Non-Cryst. Solids 71, 203 (1985).

[3] A. Meyer, J. Horbach, W. Kob, F. Kargl, and H. Schober, Phys. Rev. Lett. 93, 027801 (2004); see also A. Meyer, H. Schober, and D. B. Dingwell, Europhys. Lett. 59, 708 (2002); F. Kargl and A. Meyer, Chem. Geol. 213, 165 (2004).

[4] J. Horbach, W. Kob, and K. Binder, Phys. Rev. Lett. 88, 125502 (2002).

[5] J. Oviedo and J. F. Sanz, Phys. Rev. B 58, 9047 (1998).

[6] P. Jund, W. Kob, and R. Jullien, Phys. Rev. B 64, 134303 (2001).

[7] A. N. Cormack, J. Du, and T. R. Zeitler, Phys. Chem. Chem. Phys. 4, 3193 (2002).

[8] H. Lammert, M. Kunow, and A. Heuer, Phys. Rev. Lett. 90, 215901 (2003).

[9] W. Götze, in Liquids, Freezing and Glass Transition, edited by J. P. Hansen, D. Levesque, and J. Zinn-Justin (North Holland, Amsterdam, 1991), pp. 287-503.

[10] Th. Voigtmann, A. M. Puertas, and M. Fuchs, Phys. Rev. E 70, 061506 (2004).

[11] G. Foffi, W. Götze, F. Sciortino, P. Tartaglia, and Th. Voigtmann, Phys. Rev. E 69, 011505 (2004).

[12] M. Nauroth and W. Kob, Phys. Rev. E 55, 657 (1997).

[13] W. Kob, M. Nauroth, and F. Sciortino, J. Non-Cryst. Solids 307-310, 181 (2002).

[14] A. B. Mutiara and H. Teichler, Phys. Rev. E 64, 046133 (2001).

[15] L. Fabbian, A. Latz, R. Schilling, F. Sciortino, P. Tartaglia, and C. Theis, Phys. Rev. E 60, 5768 (1999).

[16] C. Theis, F. Sciortino, A. Latz, R. Schilling, and P. Tartaglia, Phys. Rev. E 62, 1856 (2000).

[17] S.-H. Chong and F. Sciortino, Phys. Rev. E 69, 051202 (2004).

[18] F. Sciortino and W. Kob, Phys. Rev. Lett. 86, 648 (2001).

[19] G. J. Kramer, A. J. M. de Man, and R. A. van Santen, J. Am. Chem. Soc. 64, 6435 (1991).

[20] J. Horbach and W. Kob, Philos. Mag. B 79, 1981 (1999).

[21] J. Horbach, W. Kob, and K. Binder, Chem. Geol. 174, 87 (2001).

[22] W. Götze, in Amorphous and Liquid Materials, edited by E. Lüscher, G. Fritsch, and G. Jacucci (Nijhoff Publishers, Dordrecht, 1987), vol. 118 of NATO ASI Series E, pp. 34-81.

[23] W. Götze and Th. Voigtmann, Phys. Rev. E 67, 021502 (2003).

[24] J. Horbach and W. Kob, Phys. Rev. B 60, 3169 (1999).

[25] J. Horbach and W. Kob, J. Phys.: Condens. Matter 14, 9237 (2002).

[26] J. Horbach, W. Kob, and K. Binder, J. Phys.: Condens. Matter 15, S903 (2003). 